\title{
Realignment of Slanted Fe Nanorods on Silicon Substrates by a Strong Magnetic Field
}

\author{
Yin $\mathrm{Hu}^{1}$, Zhengjun Zhang ${ }^{1}(\bowtie)$, Qin Zhou ${ }^{1}$, Wei Liu ${ }^{1}$, Zhengcao $\mathrm{Li}^{1}$, and Daqiao Meng ${ }^{2}$ \\ ${ }^{1}$ Advanced Materials Laboratory, Department of Materials Science and Engineering, Tsinghua University, Beijing 100084, China \\ ${ }^{2}$ National Key Laboratory for Surface Physics and Chemistry, Mianyang, Sichuan 621907, China \\ Received: 5 April 2010 / Revised: 21 April 2010 / Accepted: 22 April 2010 \\ (C) The Author(s) 2010. This article is published with open access at Springerlink.com
}

\begin{abstract}
Slanted Fe nanorods prepared by glancing angle deposition on silicon substrates exhibited easy magnetization along their growth axis. By using a thin gold film on a silicon substrate as a buffer layer, slanted Fe nanorods can be realigned towards the substrate surface normal by a strong magnetic field. After realignment, the Fe nanorods retained the easy magnetization axis along their growth axis. The effects of the realignment by the strong magnetic field on the properties of the slanted Fe nanorods were also investigated. This study provides a possible way to fabricate magnetic nanostructures for perpendicular recording applications.
\end{abstract}

\section{KEYWORDS}

Glancing angle deposition, Fe nanorods, easy magnetization axis, strong magnetic field, reorientation

Nanomaterials, due to their reduced size at the nanometer scale, exhibit many unique physical and chemical properties that are quite different from the bulk state, and have attracted great interest in past years [1-6]. For example, at the nanometer scale, magnetic materials can exhibit superior magnetic properties, such as higher permeability and lower coercivity, compared with their bulk forms due to the single domain configuration and nanoscale effect [7-9]. This provides a way to increase the memory density of devices by fabricating nanostructures of magnetic materials. In recent years, Fe nanostructures have attracted much attention because of their potential application in nano-devices, and several approaches have been developed to grow Fe nanostructures [10-12].

An easy way to grow Fe nanostructures is the glancing angle deposition (GLAD) technique, which has been shown to be a powerful way of fabricating $1-D$ nanostructures $[13,14]$. In this method, the morphology (such as slanted or vertically aligned nanorods, zigzags, etc.) of the final nanostructures depends on the motion of the substrate, and greatly influences the properties of the nanostructures [15-21]. We will show later in this study that slanted and vertical Fe nanorods exhibit different magnetic properties. Since arrays of magnetic nanostructures are promising candidates for novel magnetic recording media [22, 23], we may need to devise ways to adjust their orientation and/or alignment after growth.

In this letter, we report the growth of slanted and vertically aligned Fe nanorods on silicon substrates by GLAD, the easy magnetization of slanted Fe nanorods along their growth axis, and the reorientation of the slanted Fe nanorods towards the surface normal of the substrates in a strong magnetic field. The effects

Address correspondence to zjzhang@ $@$ tsinghua.edu.cn 
of the reorientation by the magnetic field on the properties of the nanorods were also investigated.

The substrates used in this study were pristine $\mathrm{Si}(001)$ wafers. These were supersonically cleaned in acetone, alcohol, and deionized water baths in sequence and then dried by a nitrogen flow. Slanted and vertical Fe nanorods were deposited on the substrate by the GLAD technique in a home-made e-beam deposition system with a vacuum level of $3 \times 10^{-5} \mathrm{~Pa}$ [14]. For the reorientation of the slanted nanorods, a gold film of $40 \mathrm{~nm}$ in thickness was deposited on the silicon substrates as a buffer layer, and the samples were sealed in quartz tubes with a vacuum level of $8 \times 10^{-5} \mathrm{~Pa}$ and annealed in a strong magnetic field (JMTD-10T150) to 200 and $250{ }^{\circ} \mathrm{C}$ for $0.5 \mathrm{~h}$. The morphology of the slanted and vertical Fe nanorods before and after annealing was examined by scanning electron microscopy (SEM, JEOL JSM 6301 working at $20 \mathrm{kV}$ ). The magnetic properties of the nanorods were evaluated using a vibrating sample magnetometer (Lakeshore 7307).

Figures 1(a) and 1(b), respectively, show SEM images of the Fe nanorods obtained by deposition with the incident flux at $85^{\circ}$ to the surface normal without substrate rotation, and with substrate rotation. Without substrate rotation, slanted Fe nanorods were deposited on the substrate, with a diameter of $\sim 20 \mathrm{~nm}$ and length of $\sim 300 \mathrm{~nm}$, whereas with substrate rotation vertical Fe nanorods with larger diameter were deposited. Figures 1(c) and 1(d) show magnetic hysteresis loops of the two samples along different directions at room temperature. The measuring magnetic fields $\boldsymbol{H}_{1}, \boldsymbol{H}_{2}$, and $\boldsymbol{H}_{3}$, were applied parallel to the surface of the film $\left(\boldsymbol{H}_{1}\right)$, parallel to the axis of the nanorods $\left(\boldsymbol{H}_{2}\right)$, and perpendicular to the surface of the film $\left(\boldsymbol{H}_{3}\right)$. It should be noted that for the vertical Fe nanorods, the film did not exhibit perpendicular magnetic properties, no matter whether the field $\boldsymbol{H}$ was parallel or perpendicular to the film. However, for the slanted Fe nanorods, the film did exhibit some anisotropic magnetization properties. When $\boldsymbol{H}$ was parallel to the growth axis of the slanted Fe nanorods, the magnetization process was much easier than when $\boldsymbol{H}$ was parallel or perpendicular to the film. This suggests

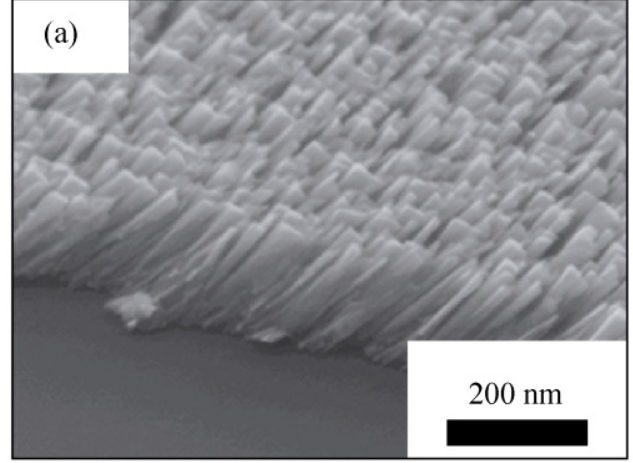

(c)

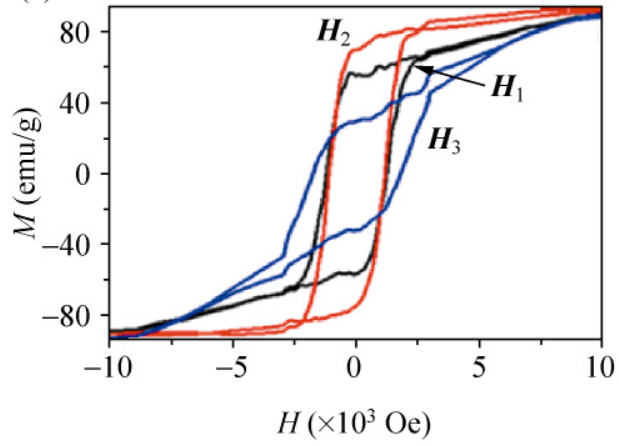

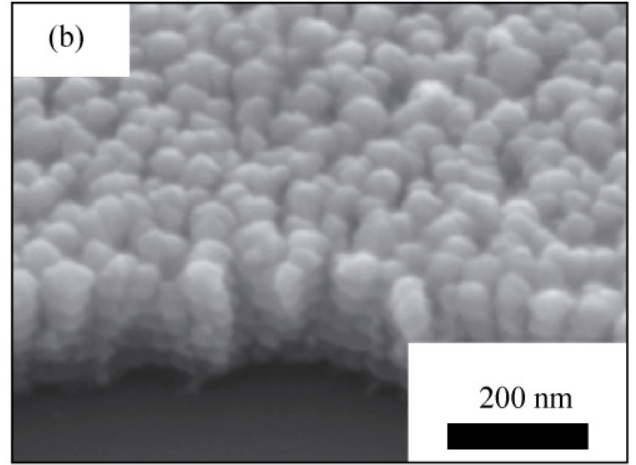

(d)

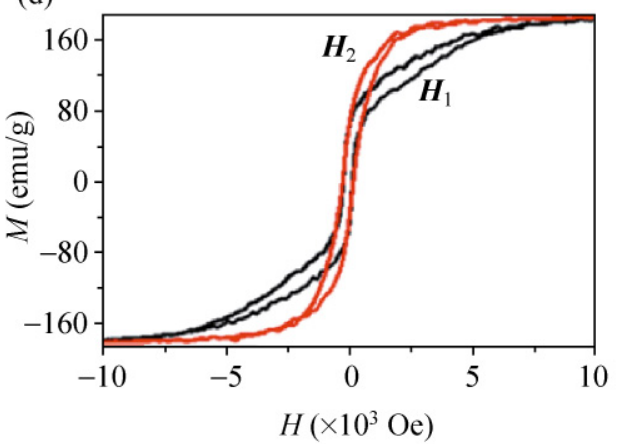

Figure 1 SEM images of (a) slanted Fe nanorods obtained by deposition at $85^{\circ}$ off the surface normal without substrate rotation, and (b) vertical nanorods deposited with substrate rotation; (c) and (d) show magnetic hysteresis loops of the two samples along different directions, i.e., $\boldsymbol{H}$ parallel to the film $\left(\boldsymbol{H}_{1}\right), \boldsymbol{H}$ parallel to the nanorod axis $\left(\boldsymbol{H}_{2}\right)$, and $\boldsymbol{H}$ perpendicular to the film $\left(\boldsymbol{H}_{3}\right)$

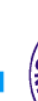


that the growth axis of the Fe nanorods is their easy magnetization axis.

If this growth axis of the nanorods were parallel to the surface normal of the substrate, these materials could be candidates for perpendicular magnetic recording applications. However, since these slanted Fe nanorods deviate from the surface normal of the substrates by large angles, it is thus necessary to find ways to reorient their growth axis (the easy magnetization axis) towards the substrate surface normal, before any applications in perpendicular magnetic recording materials are possible [24]. Naturally, a strong magnetic field [25] might be capable of doing this as these Fe nanorods are magnetic. For this purpose, we first deposited a thin gold film of thickness $40 \mathrm{~nm}$ as the buffer layer on the silicon substrate, and then deposited slanted Fe nanorods on the gold film, to make the realignment of Fe nanorods easier on the silicon substrate. We sealed the samples in quartz tubes and annealed them in a strong magnetic field of up to

\section{Fe rods grown by GLAD}

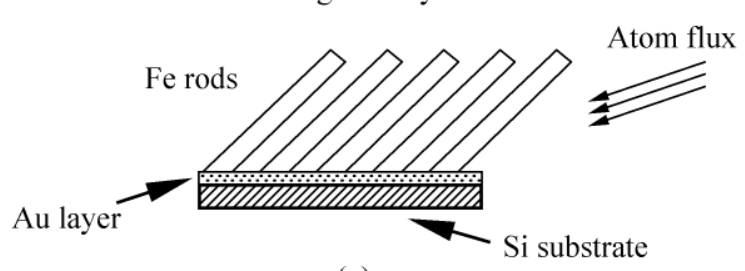

(a)

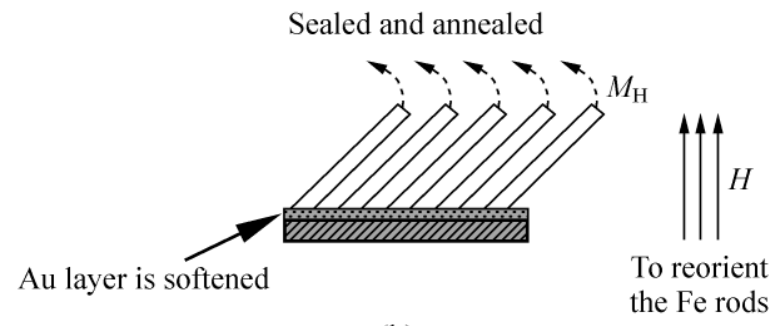

(b)

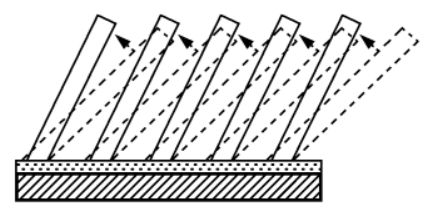

Fe rods are reoriented towards the surface normal

(c)

Figure 2 Schematic illustration of the use of a strong magnetic field to reorient the $\mathrm{Fe}$ nanorods deposited on a thin $\mathrm{Au}$ film as the buffer layer on the silicon substrate
$10 \mathrm{~T}$ at temperatures of $200{ }^{\circ} \mathrm{C}$ and $250{ }^{\circ} \mathrm{C}$ for $0.5 \mathrm{~h}$. The direction of the magnetic field was parallel to the substrate surface normal. Helped by the magnetic force, the slanted Fe nanorods should realign towards the surface normal $[26,27]$. Figure 2 shows a schematic illustration of this process.

Figures 3 (a) and 3(b) respectively show SEM images of the as-deposited slanted Fe nanorods and after

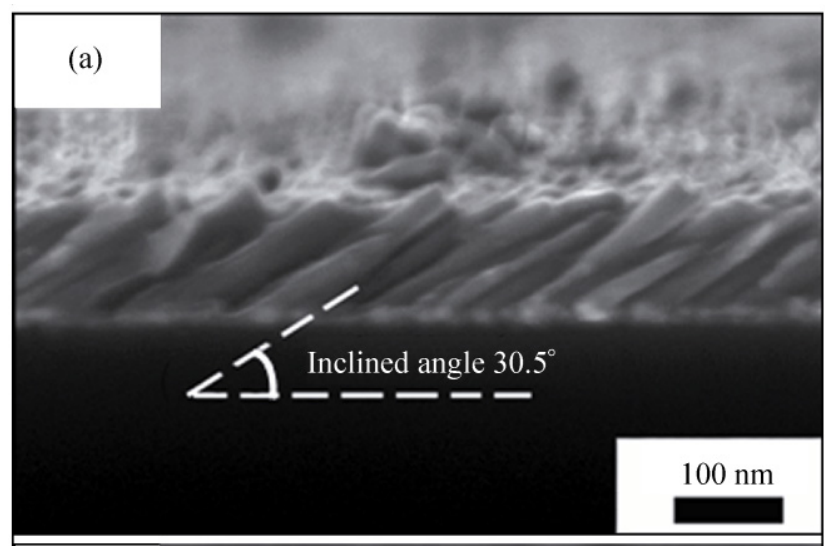

(b)

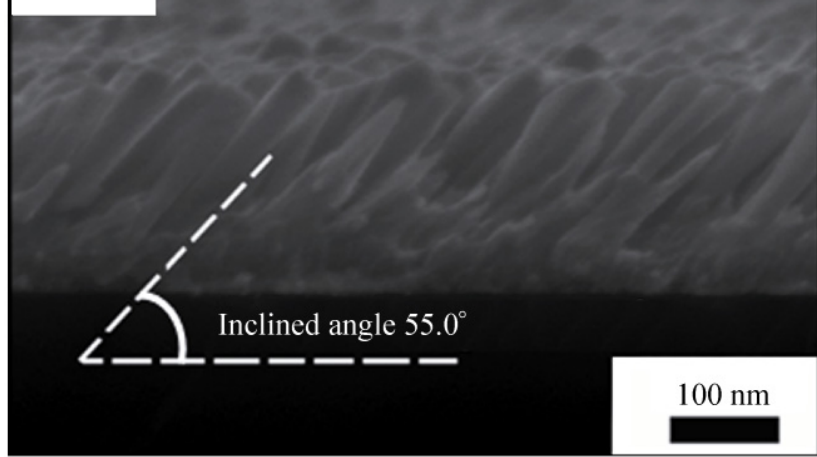

(c)

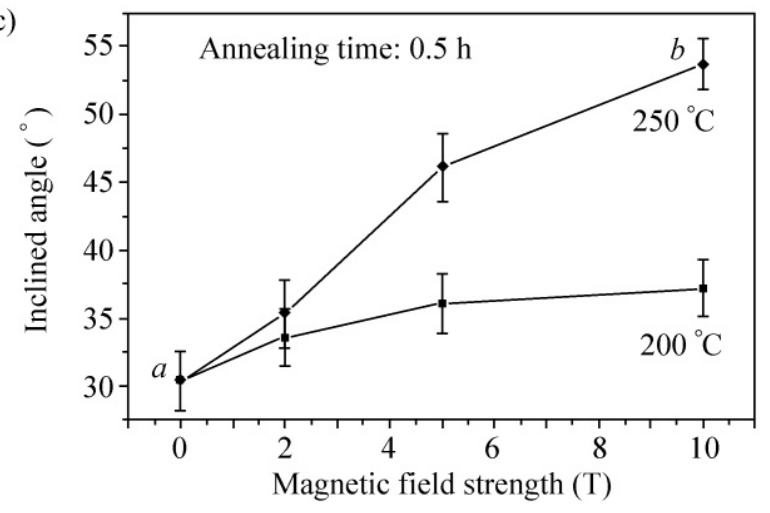

Figure 3 SEM images of (a) the as-deposited Fe nanorods and (b) after annealing at $250{ }^{\circ} \mathrm{C}$ in magnetic field of $10 \mathrm{~T}$; (c) the average angle between $\mathrm{Fe}$ nanorods and the substrate after annealing (20 Fe nanorods were counted to minimize the statistical error, which is shown as the error bar) 
annealing at $250{ }^{\circ} \mathrm{C}$ in a magnetic field of $10 \mathrm{~T}$ for $0.5 \mathrm{~h}$. The as-deposited nanorods (Fig. 3(a) are extremely slanted, with the angle between the substrate and the Fe nanorods being $30^{\circ}$. After annealing (Fig. 3(b)), the slanted nanorods are pivoted towards the surface normal, and the angle between the Fe nanorods and the substrate increases to $\sim 55^{\circ}$. In other words, the nanorods have pivoted by $\sim 25^{\circ}$. To investigate the influence of the strong magnetic field on the realignment of the Fe nanorods, we carried out the annealing experiments at two temperatures of 200 and $250{ }^{\circ} \mathrm{C}$, in magnetic fields of $2 \mathrm{~T}, 5 \mathrm{~T}$, and $10 \mathrm{~T}$. Figure $3(\mathrm{c})$ shows plots of the angle between the nanorods and the substrate after annealing under different conditions. At both annealing temperatures, the realignment is more effective at higher magnetic field, i.e., the angle between the nanorods and the substrate increases in the order $2 \mathrm{~T}<5 \mathrm{~T}<10 \mathrm{~T}$. In addition, for any given field the realignment is more obvious at $250{ }^{\circ} \mathrm{C}$ than at $200{ }^{\circ} \mathrm{C}$. This is possibly because a higher magnetic field should generate a large torque to realign the Fe nanorods, and at higher temperatures the barrier for realignment is smaller because of the lubrication of the thin Au buffer layer.

The important point is whether the easy magnetization axis after realignment in the strong magnetic field is still along the axis of the Fe nanorods. We therefore measured the magnetic hysteresis loop of the as-deposited slanted Fe nanorods (Fig. 3(a)) and that of the nanorods after annealing at $250{ }^{\circ} \mathrm{C}$ in a $10 \mathrm{~T}$ magnetic field (Fig. 3(b)). During the measurements the magnetic field $(\boldsymbol{H})$ was in the plane of the substrate surface normal and the axis of the Fe nanorods, and the angle between the substrate surface and the magnetic field $(\mathrm{H})$ was set at $0^{\circ}, 30^{\circ}, 60^{\circ}$, and $90^{\circ}$. An angle of $90^{\circ}$ means that the magnetic field is perpendicular to the substrate surface. Figures 4(a) and 4(b) respectively show the hysteresis loops measured for the two samples along different directions. It was found that for the as-deposited slanted Fe nanorods, the

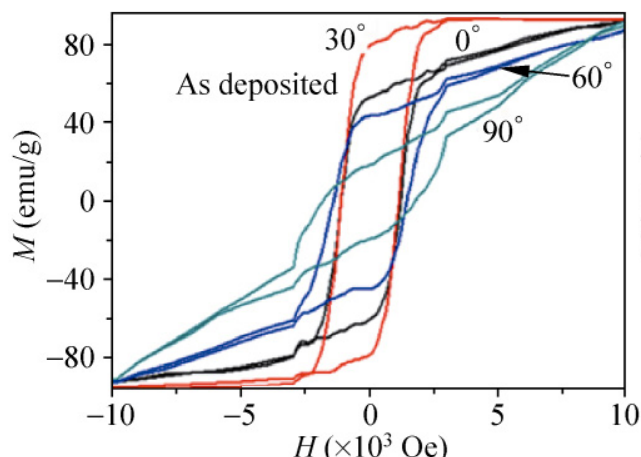

(a)

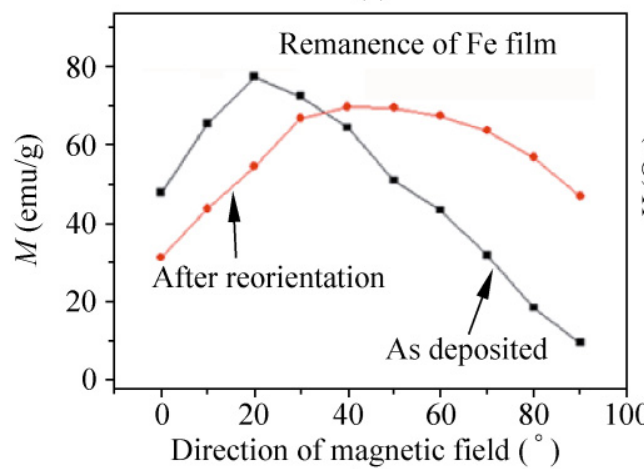

(c)

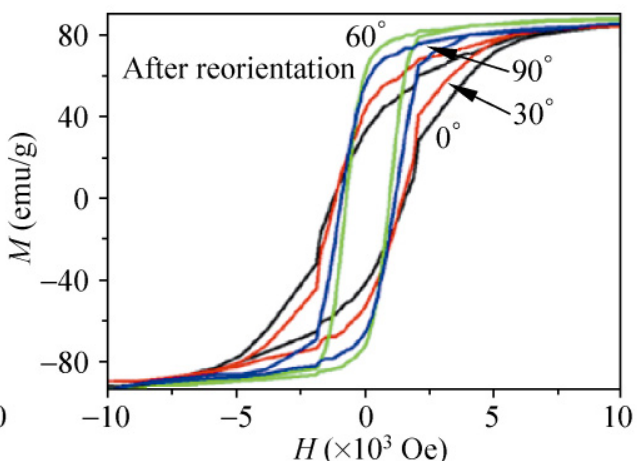

(b)

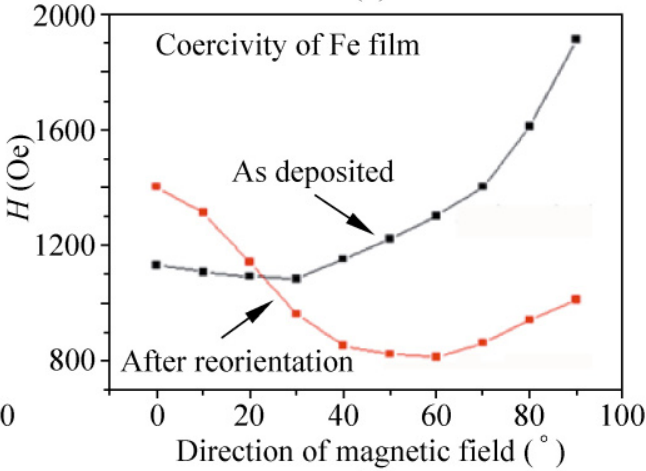

(d)

Figure 4 Magnetic hysteresis loops measured in various directions for (a) the as-deposited slanted Fe nanorods and (b) after annealing in a magnetic field of $10 \mathrm{~T}$ at $250{ }^{\circ} \mathrm{C}$; (c) and (d) plot the remanence and coercivity of the two samples, measured for various directions of the magnetic field. An angle of $90^{\circ}$ means the magnetic field is perpendicular to substrate surface 
easy magnetization axis was $\sim 30^{\circ}$ from the substrate surface, i.e., close to the axis of the Fe nanorods. It was also found that after annealing the easy magnetization axis was $\sim 60^{\circ}$ from the substrate surface, which is also close to the axis of the realigned Fe nanorods. These results indicate that the easy magnetization axis of the nanorods remains along their axis when the Fe nanorods were realigned by the magnetic field during annealing. Figures 4(c) and 4(d) show the remanence and coercivity of the two samples measured with different field directions. Both the maximum remanence and the minimum coercivity moved from angles of $\sim 30^{\circ}$ (for the as-deposited sample) to $\sim 60^{\circ}$ (for the annealed sample) from the substrate surface, confirming that the easy magnetization axis of the Fe nanorods is along their axis.

In summary, we report here the synthesis of slanted Fe nanorods whose growth axis is the easy magnetization axis, and a method to reorient these slanted nanorods towards the surface normal of the substrate by a strong magnetic field. This study might provide a way to fabricate magnetic nanostructures for perpendicular recording applications.

\section{Acknowledgements}

The authors are grateful for financial support from the National Natural Science Foundation of China (No. 50931002) and the National Basic Research Program of China (973 Program, No. 2007CB936601).

Open Access: This article is distributed under the terms of the Creative Commons Attribution Noncommercial License which permits any noncommercial use, distribution, and reproduction in any medium, provided the original author(s) and source are credited.

\section{References}

[1] Iijima, S. Helical microtubules of graphitic carbon. Nature 1991, 354, 56-58.

[2] Pan, Z. W.; Dai, Z. R.; Wang, Z. L. Nanobelts of semiconducting oxides. Science 2001, 291, 1947-1949.

[3] Law, M.; Kind, H.; Kim, F.; Messer, B.; Yang, P. Photochemical sensing of $\mathrm{NO}_{2}$ with $\mathrm{SnO}_{2}$ nanoribbon nanosensors at room temperature. Angew. Chem. Int. Ed. 2002, 41, 2405-2408

[4] Zhang, Z. J.; Zhao, Y.; Zhu, M. M. NiO films consisting of vertically aligned cone-shaped $\mathrm{NiO}$ rods. Appl. Phys. Lett. 2006, 88, 033101.

[5] Modi, A.; Koratkar, N.; Lass, E.; Wei, B. Q.; Ajayan, P. M. Miniaturized gas ionization sensors using carbon nanotubes. Nature 2003, 424, 171-174.

[6] Su, X.; Zhang, Z. J.; Zhu, M. M. Melting and optical properties of ZnO nanorods. Appl. Phys. Lett. 2006, 88, 061913.

[7] Lee, B. H.; Lee, Y. J.; Min, K. H.; Kim, D. G.; Kim, Y. D. Synthesis of nano-sized $\mathrm{Fe}-\mathrm{Co}$ alloy powders by chemical solution mixing of metal salts and hydrogen reduction (CSM-HR). Mater. Lett. 2005, 59, 3156-3159.

[8] Lee, B. H.; Ahn, B. S.; Kim, D. G.; Oh, S. T.; Jeon, H.; Ahn, J.; Kim, Y. D. Microstructure and magnetic properties of nanosized $\mathrm{Fe}-\mathrm{Co}$ alloy powders synthesized by mechanochemical and mechanical alloying process. Mater. Lett. 2003, 57, 1103-1107.

[9] Gleiter, H. Nanostructured materials: State of the art and perspectives. Nanostruct. Mater. 1995, 6, 3-14.

[10] Cui, Z. L.; Dong, L. F.; Hao, C. C. Microstructure and magnetic property of nano-Fe particles prepared by hydrogen arc plasma. Mater. Sci. Eng. A 2000, 286, 205-207.

[11] Li, F. S.; Wang, T.; Ren, L.Y.; Sun, J. R. Structure and magnetic properties of Co nanowires in self-assembled arrays. J. Phys.: Condens. Matter. 2004, 16, 8053-8060.

[12] Rougemaille, N.; Schmid, A. K. Self-organization and magnetic domain microstructure of Fe nanowire arrays. $J$. Appl. Phys. 2006, 99, 08S502.

[13] Chaney, S. B.; Shanmukh, S.; Dluhy, R. A.; Zhao, Y. P. Aligned silver nanorod arrays produce high sensitivity surfaceenhanced Raman spectroscopy substrates. Appl. Phys. Lett. 2005, 87, 031908.

[14] Zhou, Q.; Li, Z. C.; Yang, Y.; Zhang, Z. J. Arrays of aligned, single crystalline silver nanorods for trace amount detection. J. Phys. D: Appl. Phys. 2008, 41, 152007.

[15] Dick, B.; Brett, M. J. Controlled growth of periodic pillars by glancing angle deposition. J. Vac. Sci. Technol. B 2003, 21, 23-28.

[16] Zhou, C. M.; Gall, D. Branched Ta nanocolumns grown by glancing angle deposition. Appl. Phys. Lett. 2006, 88, 203117.

[17] Ni, J.; Zhu, Y.; Wang, S. H.; Li, Z. C.; Zhang, Z. J.; Wei, B. Q. Nanostructuring $\mathrm{HfO}_{2}$ thin films as antireflection coatings. J. Am. Ceram. Soc. 2009, 92, 3077-3080.

[18] Liu, F.; Umlor, M. T.; Shen, L.; Weston, J.; Eads, W.; 
Barnard, J. A.; Mankey, G. J. The growth of nanoscale structured iron films by glancing angle deposition. J. Appl. Phys. 1999, 85, 5486-5488.

[19] Hawkeye, M. M.; Brett, M. J. Glancing angle deposition: Fabrication, properties, and applications of micro- and nanostructured thin films. J. Vac. Sci. Technol. A 2007, 25, 1317-1335.

[20] Robbie, K.; Sit, J. C.; Brett, M. J. Advanced techniques for glancing angle deposition. J. Vac. Sci. Technol. B 1998, 16, 1115-1122.

[21] Smith, D. O.; Cohen, M. S.; Weiss, G. P. Oblique-incidence anisotropy in evaporated permalloy films. J. Appl. Phys. 1960, 31, 1755-1762.

[22] Martín, J. I.; Nogués, J.; Liu, K.; Vicent, J. L.; Schuller, I. K. Ordered magnetic nanostructures: Fabrication and properties. J. Magn. Magn. Mater. 2003, 256, 449-501.
[23] Liu, K.; Nogués, J.; Leighton, C.; Masuda, H.; Nishio, K.; Roshchin, I. V.; Schuller, I. K. Fabrication and thermal stability of arrays of Fe nanodots. Appl. Phys. Lett. 2009, 81, 4434-4436.

[24] Zhu, H.; Yang, S. G.; Ni, G.; Yu, D. L.; Du, Y. W. Study on magnetic property of $\mathrm{Fe}_{14} \mathrm{Ni}_{86}$ alloy nanowire array. $J$. Magn. Magn. Mater. 2001, 234, 454-458.

[25] Hu, Y.; Li, Z. C.; Zhang, Z. J.; Meng, D. Q. Effect of magnetic field on the visible light emission of $\mathrm{V}_{2} \mathrm{O}_{5}$ nanorods. Appl. Phys. Lett. 2009, 94, 103107.

[26] Goya, G. F.; Berquó, T. S.; Fonseca, F. C.; Morales, M. P. Static and dynamic magnetic properties of spherical magnetite nanoparticles. J. Appl. Phys. 2003, 94, 3520-3528.

[27] Mchenry, M. E.; Laughlin, D. E. Nano-scale materials development for future magnetic applications. Acta Mater. 2000, 48, 223-238. 